\title{
Interpretare dati, discutere e riflettere insieme: esperienze didattiche in IV e V primaria
}

\section{Interpreting data, discussing and reflecting together: teaching-learning experiences in grades 4 and 5}

\author{
Franca Ferri $^{\star}$, Francesca Martignone ${ }^{\bullet}$, Elisabetta Robotti ${ }^{\circ}$ e Cristina Sabena ${ }^{\star}$ \\ * Insegnante di scuola primaria - Italia \\ - Dipartimento di Scienze e Innovazione Tecnologica, Università del Piemonte Orientale - Italia \\ - Dipartimento di Matematica, Università di Genova - Italia \\ ^ Dipartimento di Filosofia e Scienze dell'Educazione, Università di Torino - Italia \\ 凶franca.ferri.169@gmail.com, francesca.martignone@uniupo.it, robotti@dima.unige.it, \\ cristina.sabena@unito.it
}

Sunto / In questo lavoro si presentano e analizzano due esperienze didattiche rivolte alle classi IV e V primaria, incentrate sull'interpretazione di dati e sulle loro rappresentazioni grafiche. Queste esperienze si inseriscono in un percorso più ampio che mira a costruire le basi per educare cittadini consapevoli, capaci di utilizzare strumenti scientifici per interpretare le informazioni a loro disposizione e di prendere, di conseguenza, decisioni fondate su basi solide.

Si tratta di attività contestualizzate nella normale vita di classe dei bambini, che rendono i problemi di indagine fortemente motivanti. Mostreremo come nel contesto scelto l'interpretazione di dati consenta di avviare interessanti discussioni matematiche in cui gli studenti si confrontano, generano ipotesi esplicative e producono argomentazioni.

Parole chiave: interpretazione di dati; rappresentazioni grafiche; argomentare; discussione matematica; generazione di ipotesi.
Abstract / In this work, we present and analyze two didactic experiences realized in grades 4 and 5 , focused on data interpretation and their graphic representations. These experiences are part of a wider path aimed at building the basis for educating citizens who are able to interpret the information available to them and to make sound and appropriate decisions.

These activities are contextualized in the normal classroom life of children, which make the problems of investigation strongly motivating. We will show how in the chosen context the interpretation of data allows interesting mathematical discussions in which students confront each other, formulate hypothesis and produce argumentations.

Keywords: data interpretation; graphical representations; argumentation; mathematical discussion; hypothesis generation. 


\section{Introduzione e riferimenti teorici}

La situazione di emergenza che abbiamo vissuto in questo anno di pandemia da COVID-19 (20192020) ha reso ancor più evidente la necessità di formare cittadini consapevoli che siano in grado di prendere decisioni opportune interpretando dati e informazioni, spesso forniti con rappresentazioni grafiche e descritti attraverso indici statistici. In questo articolo presentiamo due esperienze didattiche, svolte in una scuola primaria' (in IV e successivamente con gli stessi studenti in V), che riguardano la lettura e l'interpretazione di dati come punto di partenza per lo sviluppo di riflessioni e argomentazioni che coinvolgono gli alunni della classe su un problema vicino alla loro esperienza: I'interpretazione dei dati relativi a punteggi ottenuti nelle prove di verifica di matematica.

Nella scuola primaria le esperienze didattiche che riguardano la lettura e l'analisi di dati reali possono diventare dei contesti in cui gli studenti sono chiamati a riflettere per capire meglio il loro mondo. II contesto, essenziale per la statistica, ha spesso un effetto positivo sulla motivazione e sul coinvolgimento degli studenti (Gattuso \& Ottaviani, 2011). Gli studenti che ragionano su dati reali che hanno un senso per il loro vissuto diventano curiosi e spesso vanno al di là di ciò che è stato loro chiesto di fare (Kranendonk, 2006). Infatti, il loro coinvolgimento potrà essere maggiore in attività in cui si sentono in qualche modo vicini e legati ai dati che analizzano.

Nelle Indicazioni Nazionali, così come già nel documento UMI Matematica 2001, vi sono numerosi riferimenti ai contesti reali dove immergere gli studenti in situazioni complesse:

«Caratteristica della pratica matematica è la risoluzione di problemi, che devono essere intesi come questioni autentiche e significative, legate alla vita quotidiana, e non solo esercizi a carattere ripetitivo o quesiti ai quali si risponde semplicemente ricordando una definizione o una regola».

(MIUR, 2012, p. 49)

«[...] I'insegnamento della matematica deve avviare gradualmente, a partire da campi di esperienza ricchi per l'allievo, all'uso del linguaggio e del ragionamento matematico, come strumenti per l'interpretazione del reale, non unicamente come bagaglio di nozioni».

(U.M.I., 2001, p.7)

In questo modo la matematica presente nell'extrascolastico e la matematica "scolastica" non sono viste come entità separate e non comunicanti, bensì come mondi dialoganti (Gravemeijer, 1999). L'importanza della probabilità e della statistica all'interno dell'insegnamento della matematica è ormai nota, come sono ormai condivise le singole peculiarità disciplinari e i relativi punti in comune con il pensiero matematico (Ottaviani, 2008; Ben-Zvi, 2014). Educare alla lettura dei dati, così come alla loro interpretazione e utilizzo, è pertanto un obiettivo didattico fondamentale fin dalla scuola primaria. Infatti, uno dei traguardi per lo sviluppo delle competenze al termine della scuola primaria è «L'alunno [...] ricerca dati per ricavare informazioni e costruisce rappresentazioni (tabelle e grafici). Ricava informazioni anche da dati rappresentati in tabelle e grafici» (MIUR, 2012, p. 49) e negli obiettivi di apprendimento al termine della classe quinta della scuola primaria leggiamo:

«Rappresentare relazioni e dati e, in situazioni significative, utilizzare le rappresentazioni per ricavare informazioni, formulare giudizi e prendere decisioni. Usare le nozioni di frequenza, di moda e di media aritmetica, se adeguata alla tipologia dei dati a disposizione».

(MIUR, 2018, p. 51)

1. La scuola primaria in Italia dura cinque anni e corrisponde alla scuola elementare nel Canton Ticino. 
Più recentemente, nei documenti ministeriali è stato evidenziato che la statistica può essere un «efficace "cavallo di Troia" per avvicinare gli alunni alla matematica e alla sua potente capacità di spiegare e interpretare il mondo, con spirito critico e con il supporto di dati alle opinioni» (MIUR, 2018, p. 12, enfasi come nell'originale). L'introduzione di riflessioni sui dati e sugli indici statistici può quindi divenire utile veicolo per promuovere spirito critico e competenze argomentative.

Inoltre, l'analisi di dati tratti dalla realtà permette di rivisitare, arricchire di senso e consolidare in contesti concreti e motivanti anche contenuti matematici già noti agli allievi. Ad esempio, nelle esperienze che descriveremo si affronteranno attività in cui è richiesto di riflettere sul calcolo della media aritmetica, concetto già conosciuto dagli studenti coinvolti nell'esperienza. Se pensiamo ai concetti matematici come costituiti da situazioni di riferimento, schemi e rappresentazioni (Vergnaud, 1992), in questo caso il concetto di media ha come riferimento i dati relativi a punteggi numerici ottenuti proprio dagli studenti. I dati numerici, che sono anche rappresentati dalla docente in un diagramma a barre, devono poi essere manipolati attraverso schemi relativi al calcolo dei diversi indici statistici, il cui valore sarà oggetto di discussione collettiva per riflettere sul loro significato e sulle informazioni che possono dare. Facciamo qui riferimento alla discussione matematica intesa come «una polifonia di voci articolate su un oggetto matematico (concetto, problema, procedura ecc.), che costituisce un motivo dell'attività di insegnamento-apprendimento» (Bartolini Bussi, Boni \& Ferri, 1995, p. 7). Nella discussione matematica l'insegnante ha un ruolo fondamentale poiché non solo progetta una particolare discussione all'interno delle attività della classe, ma assume il ruolo di guida, quindi può influenzarla con interventi mirati. L'insegnante in questa "polifonia di voci» rappresenta la voce della cultura, e introduce quindi un punto di vista che spesso è diverso da quello degli alunni.

Le discussioni matematiche che presenteremo hanno tra gli obiettivi principali lo sviluppo di competenze di analisi critica dei dati, attraverso la generazione di ipotesi interpretative, ossia di ipotesi che danno ragione di un fatto osservato, di ipotesi progettuali, ossia di ipotesi sulle azioni da svolgere per arrivare a un certo risultato, di ipotesi euristiche, relative agli esempi scelti (Boero \& Ferrero, 1995) e delle loro giustificazioni. Si tratta di componenti molto importanti nei processi argomentativi in matematica, ma anche nelle scienze e soprattutto nell'ottica dello sviluppo di una cittadinanza attiva e consapevole: infatti ogni persona dovrebbe essere

«disponibile all'ascolto attento e critico dell'altro e a un confronto basato sul riferimento ad argomenti pertinenti e rilevanti. In particolare l'educazione all'argomentazione può costituire un antidoto contro il proliferare d'informazioni false o incontrollate».

(MIUR, 2018, p. 12)

Un cittadino non solo dovrebbe o sapere o saper riconoscere che qualcosa è vero, ma anche comprendere perché è vero e sostenere il proprio punto di vista con argomenti pertinenti, analizzando criticamente le informazioni in suo possesso. Per quanto riguarda più precipuamente la matematica, questo passaggio presuppone lo sviluppo di un approccio relazionale alla disciplina, piuttosto di uno strumentale (Skemp, 1976). Si può parlare di approccio strumentale alla matematica quando nell'insegnamento vengono principalmente proposti esercizi risolvibili con schematismi già preconfezionati e automatismi appresi senza riflessioni e consapevolezza sul perché essi sono efficaci per arrivare alla soluzione. In questo caso quindi è la memoria che ha un ruolo preponderante, non il ragionamento, e i processi risolutivi sono spesso in secondo piano rispetto alla considerazione dei risultati ottenuti. Al contrario si parla di approccio relazionale alla matematica quando il focus, durante il processo di insegnamento-apprendimento, si concentra principalmente sui processi attuati per risolvere problemi di cui spesso non si conosce a priori un algoritmo che porti alla soluzione, e in cui comunque si debbano costruire delle strategie risolutive capendo cosa fare e perché, cogliendo le relazioni strutturali tra gli enti in gioco.

Nel seguente paragrafo presenteremo brevemente un percorso didattico, svolto longitudinalmente 
su cinque anni, realizzato da una delle autrici (Franca Ferri) in una scuola primaria. II percorso sviluppa il tema dei dati e del loro trattamento in un approccio relazionale alla matematica che parte dalla classe prima per arrivare sino alla classe quinta. Nei successivi paragrafi, ci soffermeremo più in dettaglio su due specifiche attività sviluppate rispettivamente in classe IV e in classe $V$, nelle quali le riflessioni su dati e le discussioni collettive hanno lo scopo di arricchire di senso alcuni concetti matematici, analizzare le relazioni tra dati e sviluppare processi di produzione di ipotesi e argomentazioni (Sabena, Ferri, Martignone \& Robotti, 2019).

\section{Il percorso didattico}

La classe, inserita in una scuola a tempo pieno della periferia di Modena, era composta da 23 alunni di cui sei di madrelingua non italiana e un'alunna diversamente abile. Nella classe lavoravano con quantità orarie diverse quattro insegnanti: I'insegnante di italiano, I'insegnante di matematica (sempre la stessa per i cinque anni), l'insegnante di inglese e l'insegnante di sostegno (generalmente non presente durante le ore di matematica). Nella classe, nonostante i vari problemi, vi era un buon clima collaborativo e si lavorava intensamente.

Il percorso didattico è stato realizzato anche con materiali provenienti da siti ufficiali della Società Italiana di Statistica (SIS: http://www.sis-statistica.it) e dell'Istituto Nazionale di Statistica (ISTAT: http:// www.istat.it). Per comprendere a pieno il tipo di attività proposte in seguito, e situarle in un contesto didattico ampio e significativo, riportiamo ora una breve descrizione di quanto svolto dalla classe a partire dalla classe prima.

In classe prima si sono attuate semplici attività di statistica raccogliendo e organizzando dati. II tema di indagine e le modalità di raccolta dei dati erano discussi collettivamente così come la scelta della loro rappresentazione. II campo d'indagine era quasi sempre la classe di appartenenza o al massimo veniva esteso alle classi parallele. I temi di indagine erano vicini al vissuto degli alunni quali ad esempio l'animale o il cibo preferito, il numero di fratelli o sorelle, i giorni di sole, nuvole, pioggia, nebbia e neve conteggiati in un determinato mese, favorito quest'ultimo dalla registrazione quotidiana su cartellone del tempo meteorologico. Infatti, in un primo tempo i grafici su cartellone erano costruiti con rappresentazioni iconiche dei dati: su un cartoncino si disegnava il simbolo di rappresentazione del tempo atmosferico del giorno e i cartoncini venivano poi incollati sul cartellone come componenti di un diagramma. Successivamente si è passati all'introduzione di diversi simboli per rappresentare i dati. II significato dei simboli era riportato in apposita legenda (un percorso simile per la classe prima è "L'animale preferito" nel progetto M@t.abel, http://www.scuolavalore.indire.it/nuove risorse/lanimale-preferito/).

In classe seconda il lavoro si è concentrato sulla costruzione di vari diagrammi a barre e tabelle di frequenza al fine di mostrare diverse rappresentazioni di uno stesso oggetto (insieme di dati). Per esempio, durante l'intero anno scolastico, e anche negli anni successivi, si sono raccolte e registrate giornalmente le temperature esterne. La raccolta di questi dati abituava così gli alunni all'osservazione di una massa di informazioni (dati) ottenute in condizioni analoghe (alla stessa ora, 8:45, sempre nello stesso luogo e con lo stesso termometro). Dai dati raccolti ogni mese si costruiva poi un grafico sulla base del quale si avviava un confronto e si sviluppavano riflessioni: «Ad ogni giorno corrisponde un pallino che indica i gradi di quel giorno. Ad esempio, l'8 febbraio c'erano $-3^{\circ} \mathrm{C} »-$ «Per quattro giorni ci sono stati $-4^{\circ} \mathrm{C}$ : il 10 , I' 11 , il 12 e il 15 febbraio» - «Febbraio è stato molto più freddo di gennaio perché in gennaio si è andati solo una volta sotto zero».

Poco alla volta, I'attività didattica è passata dalla costruzione dei grafici e diagrammi all'interpretazione di questi. Sono stati quindi presentati agli alunni diversi grafici, anche non legati direttamente al 
loro vissuto e con diversi tipi di scale.

In classe terza si sono introdotti gli indici statistici moda e media aritmetica. In particolare, l'introduzione della media è avvenuta attraverso una discussione collettiva: I'insegnante, consegnando ad ogni alunno il diagramma che rappresentava i punteggi ottenuti dagli alunni della classe in una prova di verifica, aveva riportato anche la media dei punteggi e aveva aperto la discussione chiedendo: "Quando vi ho dato il grafico dei vostri punteggi vi ho scritto che la media dei punteggi della classe IIIC era di 8,27. Secondo voi cos'è la media? Come si ottiene?». La domanda, che punta sulla costruzione del significato dello strumento matematico, ha fatto emergere interessanti concezioni basate sulle esperienze quotidiane degli allievi. Fra queste, per esempio:

\footnotetext{
«Per me la media è come per le scuole: la scuola media è normale, invece le elementari sono facili e le superiori difficili. Ė quella cosa che sta in mezzo. Per avere la media devi aggiungere qualcosa a quello che è facile e devi togliere qualcosa a quello che è difficile. Anche con i punteggi: si aggiunge qualcosa a chi ha un punteggio basso e si toglie qualcosa a chi ha un punteggio alto». «Per me la media è circa l'equilibrio tra tutti i numeri che abbiamo».

«La media è circa la metà dei punteggi che abbiamo ottenuto. Si vede bene al computer se fai un grafico e vedi che il punteggio medio è verso la metà».

«La media è circa la metà. È dove i punteggi è come che si fermino, cioè che sono tutti concentrati lì. Si ottiene facendo circa la metà dei punteggi. Li sommi tutti e poi fai diviso 2 ».

«Per me la media si ottiene quando ci sono dei numeri. Se non ci sono dei numeri non puoi parlare di media. È una cosa che riguarda la matematica».
}

A partire dalle ipotesi interpretative e progettuali emerse nella discussione, non solo si è arrivati a definire il significato di media, ma si è definita anche la corretta procedura per calcolarla. La discussione e la riflessione si è estesa anche agli altri indici statistici.

In classe quarta è stata oggetto di discussione e di definizione la procedura per individuare la mediana e si sono messi in relazione i tre indici statistici in situazioni problema proposte dall'insegnante e con domande stimolo per guidare verso la costruzione del sapere in un approccio relazionale alla matematica.

In classe quinta si è continuato a presentare problemi che richiedevano il calcolo degli indici statistici. Si sono presentate anche attività per sviluppare riflessioni sulle proprietà degli indici e sulle relazioni tra i numeri. Nel corso dell'anno sono state considerate situazioni e contesti diversi, raccogliendo dati dalle diverse discipline quali, ad esempio, la geografia e la storia, al fine di osservare fenomeni economici e sociali e motivare gli alunni alla soluzione dei compiti.

\section{Un'esperienza didattica in classe IV}

In questo paragrafo presentiamo più in dettaglio alcuni estratti dell'attività svolta in classe IV a partire dai dati numerici ottenuti dagli studenti come punteggi in prove d'ingresso o verifiche quadrimestrali. II contesto dei punteggi ottenuti in prove di verifica coinvolge gli alunni in prima persona: ciò viene sfruttato dall'insegnante per promuovere riflessioni e confronti sui dati raccolti, attraverso mirate discussioni matematiche. Qui l'insegnante gioca il ruolo chiave di guida verso la costruzione della conoscenza.

La scelta di far lavorare i ragazzi sui punteggi ottenuti nella prova di verifica quadrimestrale - dati, quindi, che li coinvolgono direttamente - è dovuta essenzialmente a due motivazioni di carattere diverso. Un primo motivo riguarda la didattica della statistica: lavorare su dati raccolti dalla classe interpretandoli, porta a una maggiore consapevolezza della necessità di riflettere sui dati per formarsi 
delle opinioni. L'altro motivo è di carattere più pedagogico e riguarda la scelta di lavorare "pubblicamente" sui punteggi ottenuti dai ragazzi in una prova. In questo modo il tabù della secretazione del punteggio ottenuto, quasi sempre rigorosamente celato, cade e il punteggio diviene oggetto di confronto, di riflessione e di consapevolezza del proprio risultato e «dei risultati della classe, in una prospettiva in cui la valutazione assume la funzione formativa di accompagnamento dei processi di apprendimento e di stimolo al miglioramento continuo» (MIUR, 2012, p. 13).

Dopo aver corretto, assegnato il punteggio e consegnato la prova di verifica del primo quadrimestre, I'insegnante consegna ad ogni alunno una scheda (Figura 1) in cui è riportato un grafico che rappresenta l'ordinamento dei punteggi delle prove di verifica del primo quadrimestre. Ogni colonna del grafico rappresenta uno studente della classe (23 in totale) e la media dei punteggi ottenuti nella verifica quadrimestrale composta da più domande inerenti ai vari nuclei della matematica.

Il punteggio che ho ottenuto nelle prove di verifica quadrimestrali di matematica è di ...... su 10.

1. Nel grafico riportato sotto colora la colonna corrispondente al tuo punteggio.

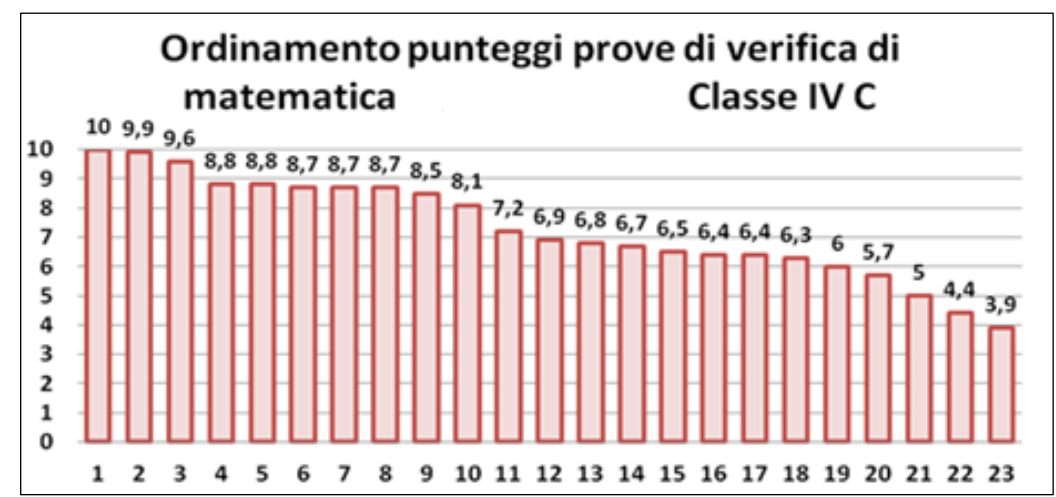

2. Osserva ora questi dati riferiti alla classe e completa le frasi:

- Media aritmetica: 7,3

- Mediana: 6,9

- Moda: 8,7

Per ottenere la media aritmetica io ho

Per individuare la mediana io ho

Per individuare la moda io ho

3. Ora, osservando media, mediana, moda e il tuo punteggio, scrivi alcune osservazioni/considerazioni. 
Osserviamo che il problema d'indagine, cioè cosa si vuole conoscere in questo compito, così come i dati considerati per l'indagine stessa, sono definiti dall'insegnante e gli alunni non sono direttamente coinvolti in questa scelta. II contesto specifico del problema però è legato al vissuto degli alunni e le modalità della rilevazione, in particolare i dati raccolti cioè i punteggi, e le modalità di tale raccolta derivano dall'osservazione diretta e definiscono le caratteristiche di concretezza che genera motivazione alla soluzione del compito.

In generale, si tratta qui di interpretare un diagramma e non di costruirlo (la raccolta dei dati e la rappresentazione è effettuata dall'insegnante). Osserviamo che, nonostante l'aspetto, il diagramma proposto non è un istogramma, perché non rappresenta frequenze, ma riporta il valore assunto da ogni dato. I dati sono quantitativi e pertanto è possibile determinare gli indici statistici, così come fatto dall'insegnante.

In particolare, questo compito prevede più consegne: nella prima consegna l'insegnante richiede che ogni alunno identifichi la colonna del grafico con il punteggio da lui ottenuto allo scopo di avere una prima percezione visiva della posizione del proprio punteggio all'interno di quelli della classe. In questo modo gli allievi iniziano a confrontare in modo percettivo il proprio punteggio con la sua posizione all'interno della classe. In alcuni casi, la visualizzazione nel diagramma della posizione della colonna corrispondente al proprio punteggio induce gli allievi a un confronto con la realtà della classe e a riconsiderare quindi il proprio grado di soddisfazione, come nel caso di An. : «lo, che ho un punteggio di 6,4, pensavo di essere andato abbastanza bene ed ero contento, ma se lo guardo qua vedo che è un punteggio abbastanza bassino e sono meno contento».

Nella seconda consegna l'insegnante fornisce gli indici statistici da lei elaborati e chiede agli alunni di scrivere la procedura che attuerebbero per ottenere quei dati numerici. Si tratta di una consegna che ha lo scopo di identificare incertezze o lacune legate alle procedure di calcolo degli indici per intervenire didatticamente. La consegna, infatti, è caratterizzata dalla richiesta meno usuale di identificare la procedura a partire da un risultato. Per spiegare come ottenere la media aritmetica vi è chi descrive esattamente la procedura, specificando anche il ricorso a una calcolatrice, vista la "lunghezza" dell'addizione, come scrive Or.: «... preso una calcolatrice, sennò si impazziva a fare un'addizione così lunga, sommato tutti i punteggi, poi la somma I'hai divisa per 23. Credo sempre con la calcolatrice»; chi, invece, descrive molto sommariamente la procedura lasciando dei dubbi sul reale livello di competenza: «... hai sommato tutti i numeri poi li hai divisi e così ti usciva la media». Osserviamo come le risposte degli alunni identifichino chiaramente l'insegnante come esecutrice della procedura, interpretando quindi la frase della consegna: "Per ottenere la... io ho..." come la richiesta di formulazione di un'ipotesi progettuale per il calcolo degli indici. Soprattutto per quanto riguarda la procedura per individuare la mediana molti allievi hanno scritto semplicemente «... hai preso il valore che sta in mezzo» tralasciando la necessità dell'ordinamento dei punteggi. Questo fatto ha indotto I'insegnante a rivedere insieme agli allievi la procedura per individuare la mediana, anche mediante altri esempi. In questo modo si può osservare come l'insegnante, nel processo di avvio e conduzione della discussione collettiva, guida la revisione della formulazione di ipotesi interpretative e progettuali e, tramite esempi mirati, supporta la giustificazione e la costruzione di senso per le procedure di calcolo adottate.

La terza consegna vuole sollecitare gli allievi a riflessioni di confronto fra il proprio punteggio e gli indici statistici ottenuti. Come sappiamo, infatti, la rappresentazione dei dati sul grafico permette di cogliere visivamente alcuni aspetti del fenomeno in questione (il suo andamento ecc.) ma, per andare al di là della "percezione", occorre tradurre i dati in valori che permettano di valutare rapidamente il fenomeno e, per questo, si usano gli indici statistici e si dà avvio alle riflessioni. Di seguito riportiamo degli esempi che abbiamo selezionato per mostrare alcune riflessioni su aspetti di tipo relazionale:

- riflessioni sul proprio punteggio rispetto agli indici statistici:

Pi.: «ll mio punteggio è inferiore alla media, alla mediana e alla moda. Sono andato abbastanza bene, anche perché non ho preso meno di 6 , che era il mio obiettivo». 
Di fatto si osserva un semplice confronto dei valori corrispondenti al punteggio ottenuto, agli indici statistici e al punteggio corrispondente alla sufficienza;

- riflessioni sul proprio punteggio rispetto alla significatività degli indici statistici:

Fi.: «ll mio punteggio è superiore alla mediana e alla media, ma è inferiore alla moda. La moda secondo me è un po' per caso che è di 8,7 , perché poteva anche essere di 6,4 o di 8,8 . Sono andato bene, sono contento».

Si intuisce qui un approccio all'analisi delle frequenze. In un certo senso, infatti, Fi. identifica nel grafico tre classi $(8,7,6,4$ e 8,8) con (quasi) la stessa frequenza (2 o 3 occorrenze).

An.: «ll mio punteggio di 7,2 è vicinissimo alla media e io sono contenta, anche se il punteggio della media in realtà non esiste, perché nessuno di noi ha preso 7,3. Questa per me è una cosa strana».

Qui si osserva una riflessione sulla relazione fra i valori degli indici (in questo caso, la media) e i dati rilevati (in questo caso, la media dei punteggi per alunno) che non sempre compaiono contemporaneamente nei due insiemi (quello dei valori degli indici e quello dei dati rilevati).

- riflessioni sull'andamento della classe in base agli indici statistici:

Fe.: "Secondo me la media è quella che dà più l'idea di come è andata la classe. Se qualcuno ti chiede come è andata la verifica di matematica, tu puoi dire abbastanza bene perché la media è di 7». Osserviamo come l'interpretazione degli indici statistici consenta a Fe. di sviluppare un'analisi sull'andamento generale del fenomeno proponendo quindi una visione d'insieme.

Au.: «La media della classe è 7,3, quindi siamo andati abbastanza bene. La mediana è 6,9 , che è vicina al 7, quindi quasi come la media. Possiamo dire che 11 bimbi hanno preso più di 7 e 11 bimbi hanno preso meno di 7».

Osserviamo come Au., mettendo in relazione media e mediana e la loro vicinanza, intuisce una distribuzione asimmetrica dei dati. Au., infatti, non aggiunge alcuna considerazione sulla moda, forse perché più distante dai precedenti valori. Inoltre, anche in questo caso viene proposta una visione d'insieme: questa considerazione, infatti, lascia intendere come, grazie all'interpretazione del grafico su dati continui (media dei voti dei bambini), Au. riesca a visualizzare un raggruppamento in classi (voto $>7$; voto $<7$ ) che gli consente, appunto, di interpretare l'andamento dei punteggi delle prove di verifica di tutti i compagni. L'analisi dell'andamento generale del fenomeno, e quindi di una visione d'insieme, costituisce un momento di consapevolezza collettiva (come va la classe) che può essere messa in relazione alla consapevolezza individuale (come va il singolo) e che costituisce un utile elemento di avvio alla discussione di classe che può porre le basi anche per lo sviluppo di una valutazione formativa;

- riflessioni che riguardano l'interpretazione del grafico, da cui seguono inferenze:

Ma.: «Se tu prendi il punteggio massimo che è 10 e quello minimo che è 3,9, poi li dividi per due ottieni 6,9, insomma quasi 7, e anche questo si avvicina alla media vera e allora per farti un'idea di come va, puoi anche prendere il minimo e il massimo e poi dividerli per $2 »$. Anche in questo caso l'analisi dell'andamento del fenomeno è basata sull'analisi dei dati statistici e della loro relazione e si percepisce qui una prima intuizione sugli indici di variabilità. Ancora, la riflessione è centrata sull'andamento generale della classe. Questo è probabilmente il frutto della pratica della discussione collettiva avviata dall'insegnante fin dalla classe prima.

Come abbiamo visto, quindi, il focus di questa attività non è solo la revisione e l'applicazione delle procedure per ottenere gli indici indicati, ma soprattutto la riflessione sul significato di tali indici e sulle informazioni che essi ci possono dare rispetto al fenomeno da osservare, secondo un approccio relazionale alla matematica. Non si tratta solo di conoscere e saper applicare delle procedure, ma di comprenderne il significato e il risultato nel contesto in cui queste sono utilizzate. Per questo, la struttura della consegna segue un ordine ben preciso che va dal manipolare i dati all'interpretarli riflettendo sulle informazioni che possono fornire in relazione a scopi, aspettative e conoscenze. Tutto 
questo ha l'obiettivo di andare oltre il piano delle conoscenze e delle abilità e di favorire lo sviluppo di competenze che portino a un saper agire riflessivo e consapevole in situazioni analoghe e di possedere esperienze potenzialmente utili per affrontare problemi aperti, come quello proposto nella seguente attività.

\section{Un'esperienza didattica in classe $\mathrm{V}$}

Nella stessa classe, l'anno successivo, all'inizio della quinta, dopo la prova d'ingresso di matematica, l'insegnante ha dato la seguente consegna individuale:

La media aritmetica dei punteggi della classe $V \mathrm{C}$ nelle prove d'ingresso è stata di 6,83 . Cosa sarebbe necessario fare per alzare la media di un punto ed avere così un punteggio di 7,83 ?

Questa consegna vuole sviluppare riflessioni sulle proprietà della media aritmetica e sulle relazioni tra i numeri, sulle operazioni che portano alla generazione o variazione del valore della media. Qui il focus è sulla generazione di ipotesi sulle possibili azioni che portano a quel risultato, e sulla successiva giustificazione di queste attraverso l'analisi delle relazioni tra i numeri e le operazioni coinvolte nella procedura di produzione della media. Per questo, il problema non richiede solo una conoscenza e un'applicazione di procedure. Si tratta di un primo approccio verso problemi in cui è richiesto di immaginare e di manipolare delle relazioni conoscendo i risultati finali, le operazioni coinvolte nella procedura che li ha generati e i numeri che possono variare in un intervallo definito (anch'esso conosciuto e familiare agli alunni), non conoscendo però la specifica sequenza di numeri.

II problema può essere affrontato con diverse strategie risolutive, corrette o meno, che saranno poi oggetto di discussione con la classe.

L'insegnante raccoglie le risposte degli allievi e ne seleziona tre da proporre in una discussione collettiva. La scelta di queste tre risposte è rappresentativa di ciò che è emerso nella classe e, per questo, fra le risposte selezionate, potranno essercene alcune errate. La scelta è dovuta anche a esigenze comunicative: infatti, queste risposte possono prestarsi bene a un'analisi da parte degli alunni, essendo espresse in un linguaggio sintetico e chiaro. L'insegnante trascrive alla lavagna le tre risposte scelte e apre la discussione:

1. Mi.: «È sufficiente aggiungere 1 ad un punteggio, ad esempio al mio che è anche basso, e si avrebbe una media più alta».

2. Fe.: «È necessario aggiungere 23 al punteggio totale per avere la media più alta di 1 punto».

3. Ou.: «Basta aggiungere 10, che era il punteggio massimo che si poteva avere, per avere la media più alta».

Di seguito riportiamo gli interventi più salienti della discussione di classe e, per ciascuno di essi, un commento critico.

Ins.: "Analizziamo ora i tre ragionamenti e vediamo chi ha ragione e perché. Vi prego di intervenire dopo aver ben pensato e di motivare i vostri interventi».

La richiesta è esplicita: analizzare le tre diverse risposte e vedere quali sono corrette e quali no. Da come è posta la consegna, è implicito che almeno una di queste sia corretta e che ce ne siano anche di non corrette. L'invito agli allievi è alla riflessione e all'argomentazione, richieste fondamentali nell'approccio didattico seguito dall'insegnante. 
Ma.: «Ci puoi dire quanto era il punteggio totale, così possiamo verificare con i conti?»

L'alunno richiede di avere maggiori informazioni (numeriche), verosimilmente per cercare di applicare le procedure conosciute per il calcolo della media al fine di modificarne il valore: conoscendo il numero degli alunni, serve almeno la somma totale dei punteggi per procedere in modo empirico. Si potrebbero infatti calcolare le medie secondo le tre proposte messe in discussione. L'approccio quindi è procedurale e l'allievo sembra non formulare ipotesi.

Ins.: «Credi sia necessario? Hai molta fretta di sapere se le proposte sono corrette? Prova a riflettere. Comunque, la somma di tutti i punteggi era 157,176».

L'insegnante invita l'alunno a non affidarsi esclusivamente ai dati numerici ma a riflettere sulle relazioni che legano tali dati; lo invita inoltre a riflettere sulle soluzioni proposte per capire che si potrebbe procedere anche senza esplicitare questo dato. In altre parole, I'insegnante invita l'alunno a produrre delle ipotesi prima di avviare le procedure di calcolo. Poi però gli fornisce il numero richiesto (decisione motivata da variabili che esulano dal focus dell'articolo).

Fi.: «Si capisce anche senza fare dei conti. Se tu aggiungi 1 non puoi avere un punto in più di media per tutti. 1 lo devi dividere per 23 e il punteggio sarà più alto di $0,0 \ldots$.

L'alunno produce dapprima un'ipotesi interpretativa descrivendo il perché il risultato non corrisponde alla richiesta (la media non diventa 7,83) e successivamente produce un'ipotesi procedurale descrivendo le operazioni da svolgere per arrivare al risultato auspicato. Cerca di rispondere ragionando sulle operazioni, sulle relazioni numeriche e sui numeri coinvolti senza usare il dato appena richiesto dal compagno: afferma infatti che non sono necessari calcoli per capire che la proposta di Mi. non è corretta poiché aggiungendo 1 alla somma dei punteggi, chiaramente non si avrà un punto in più per tutti perché l'uno andrebbe diviso tra i 23 alunni. Nella formulazione della sua ipotesi procedurale (aggiungere 23 inteso come $23 \times 1$ ) il suo ragionamento è di avvio alla generalizzazione.

Au.: «Infatti la Mi. non dice che aumenta di uno la media, ma che la media diventa più alta. Anche Ou. dice così. E se aggiungi 1 o 10 la media diventa più alta».

In questo caso l'alunno fa un'analisi accurata delle affermazioni in discussione, notando che in effetti le due compagne hanno scritto che la media diventerà più alta, non che aumenterà di un punto, quindi, a suo parere, sono affermazioni corrette. Osserviamo qui come nella discussione matematica giochi un ruolo essenziale sia l'analisi delle ipotesi generate dalle diverse voci, sia il loro confronto.

Fi.: «Sì, ma non di 1 ».

Fi. ribadisce l'inesattezza delle soluzioni che propongono di aggiungere 1 o 10 perché non conformi alla richiesta: aumentare la media proprio di 1 e non semplicemente aumentarla.

Au.: «Ok, hanno sbagliato, ma non hanno scritto male del tutto. Forse non hanno ben capito cosa ci chiedevi».

Nel suo tentativo di immedesimarsi nelle compagne per capirne il ragionamento, Au. ribadisce la distinzione fra analisi del testo della consegna e correttezza delle affermazioni delle compagne. Si identifica il problema nell'obiettivo della consegna.

Ou.: «lo ho capito cosa ci chiedevi e ho pensato che se aggiungevo 10, che era il massimo del punteggio, forse la media diventava più alta di uno. Più di dieci non potevo aggiungere, perché più di dieci non lo poteva prendere nessuno. Non ho capito perché la Fe. ha aggiunto 23. II punteggio 23 non esiste».

L'alunna giustifica la scelta di aggiungere 10 con il fatto che 10 è il punteggio massimo ottenibile. 
Dichiara infatti, rimanendo nel contesto dei punteggi, di non comprendere il 23 aggiunto dalla compagna, poiché tale numero non fa parte dei possibili valori, che vanno da 0 a 10, utilizzabili per la valutazione degli elaborati degli alunni. Sono assegnati allora due significati ai valori numerici: il valore in quanto valutazione e il dato come entità partecipante della relazione numerica. Sembra mancare la padronanza delle relazioni tra le quantità in gioco: la quantità ottenuta dai valori che partecipano alla somma e riguardano il sistema valutativo dell'insegnante, e i valori che consentono di manipolare la relazione tra le quantità in gioco. II fatto di usare un numero che non appartiene ai possibili voti non rientra nello schema che definisce la procedura per ottenere la media e quindi è difficile comprendere come usarlo per manipolare la relazione.

Fe.: «Noi siamo in 23 e io ho immaginato che tutti noi avevamo preso un punto in più e allora ho fatto $23 \times 1=23$ e l'ho aggiunto e così la media diventava 7,83».

L'allieva argomenta la procedura seguita giustificando la scelta di aggiungere 23: ha immaginato di distribuire un punto in più a tutti i voti dei 23 allievi senza curarsi di quali fossero effettivamente, ma verificando che l'aggiunta di 23 alla somma totale facesse alzare di uno la media. Come lo abbia verificato, però, non è esplicitato. Si intuisce qui l'avvio a un processo di generalizzazione perché il ragionamento è svincolato dai valori numerici associati ai voti e viene centrato sulla relazione di questi alla media. Si può quindi identificare all'interno della procedura descritta da Fe. un'ipotesi euristica legata alla scelta di particolari valori su cui operare, nello specifico aggiungere 1 a tutti i possibili voti.

Ou.: «Sì, ma Chri. e Fa., che hanno preso 10 non possono prendere un punto in più perché 10 era il massimo. Per me hai sbagliato».

L'alunna ribadisce un'informazione importante legata a considerazioni sul contesto reale della classe e dei valori numerici intesi come punteggi di ciascun alunno. La sua affermazione è infatti corretta e vuole mostrare come il ragionamento della compagna porta al risultato giusto, ma con una motivazione che non vale sempre. Qui emergono aspetti legati alla matematica come strumento di generalizzazione dell'evento reale (Fe.) e come strumento per interpretare la realtà (Ou.).

Or.: «Ou. tu non devi pensare ai bambini veri e ai punteggi che abbiamo preso, ma devi ragionare più in generale. Ti faccio un esempio facile. Se il punteggio totale fosse stato 46 e noi siamo in 23 , la media sarebbe stata di 2. Se tu a 46 aggiungi 23 ottieni 69 e 69 diviso 23 fa 3 e sarebbe un punto in più di media. Se a 46 aggiungi 10, ti viene 56 e 56 diviso 23 non fa 3. Poi volevo anche dire che la Mi. aggiungendo 1 ha pensato solo a lei e non al problema generale. Lo dice anche».

L'idea di Or. è centrata sul duplice significato dei valori numerici: valore in quanto valutazione/ punteggio e valore come dato partecipante della relazione numerica fra punteggi. Questi esempi empirici possono essere letti come controesempi all'interno della sua argomentazione, per far comprendere perché aggiungere 1 o 10 non va bene, in una tensione verso il "ragionare più in generale".

Ma.: «lo con la calcolatrice ho fatto $(157,176+1): 23$ e fa 6,87 e non va bene, perché ci sono solo 4 centesimi in più. Poi ho fatto $(157,176+10): 23$ e fa 7,26 e anche questo non va bene. Poi ho fatto $(157,176+23): 23$ che va bene perché fa 7,83 che è un punto in più di 6,83. La Fe. aveva ragione». Interviene a questo punto l'alunno che ha chiesto il valore della somma dei punteggi, e che ha verificato la correttezza delle affermazioni delle tre compagne attraverso l'applicazione diretta della procedura per trovare la media (usando cioè una prova empirica). II calcolo diventa in questo modo una prova per la classe e ha lo scopo di convincere che la proposta di aggiungere 23 sia la soluzione corretta al compito. 
Il lavoro di esplorazione e analisi delle proprietà della media aritmetica e delle relazioni tra gli elementi che la definiscono può naturalmente proseguire e, in un'ottica verticale e di costruzione a spirale del sapere, essere ripreso nella scuola secondaria ${ }^{2}$ utilizzando formule e trasformazioni algebriche per gestire le relazioni tra le diverse variabili.

Infine, notiamo che oltre alle questioni relative ai concetti matematici in gioco, nell'estratto di discussione riportato si evidenziano le competenze trasversali mostrate dagli alunni nella comunicazione e nel confronto tra pari e con l'insegnante: gli alunni infatti cercano di capire il pensiero altrui, si pongono domande e argomentano sulla verità o meno di affermazioni portando esempi e controesempi, in linea, cioè, con le Indicazioni Nazionali.

\section{Riflessioni finali}

Le attività proposte nel percorso didattico descritto, oltre a stimolare l'analisi di dati tratti da situazioni reali e ad attivare un generale spirito critico, hanno permesso di arricchire di senso e consolidare diversi contenuti matematici e hanno avviato riflessioni sulla generalizzazione della relazione fra i dati stessi. Per esempio, nell'interpretazione e nello studio del fenomeno legato alla votazione finale delle prove di matematica, è stato chiesto agli alunni di interpretare il grafico e di interpretare anche la scelta di tale grafico e della sua organizzazione (ricordiamo che esso non rappresentava la frequenza dei dati ma ne ha stimolato una lettura e l'interpretazione). Una sorta di meta analisi sull'uso delle rappresentazioni e sul loro ruolo nell'interpretazione dei fenomeni.

L'attività di IV primaria, lasciando libertà nell'interpretazione degli indici statistici agli alunni della classe, consente di attivare diversi approcci di analisi perché ciascun alunno è stimolato da domande di interesse personale diverso. Questo spinge il coinvolgimento e la motivazione dell'alunno al perseguimento della soluzione del compito, il quale diviene un compito non solo agito ma anche partecipato. Come si evince dall'analisi della discussione, essa gioca un ruolo chiave per avviare l'interpretazione dei dati e delle relazioni numeriche tramite la formulazione di ipotesi. Per questo, le ipotesi espresse dagli studenti vanno qui intese come congetture per spiegare i fenomeni mostrati dai dati e dagli indici statistici. La discussione, infatti, consente l'avvio al confronto delle ipotesi sia legate all'interpretazione dei dati (riflessioni sul proprio punteggio rispetto agli indici statistici e riflessioni sul proprio punteggio rispetto alla significatività degli indici statistici), sia allo sviluppo di progetti d'azione (riflessioni sull'andamento della classe in base agli indici statistici). Ciò sembra consolidare la costruzione dei significati degli indici statistici e, quindi, I'apprendimento negli alunni della classe. Infatti, le inferenze che seguono l'interpretazione del grafico portano la riflessione sull'andamento generale della classe spostando quindi il focus di interesse da riflessioni di tipo locale a riflessioni di tipo generale. Nell'attività proposta in $\vee$ primaria, osserviamo come le modalità di verifica delle ipotesi prodotte assumano diverse caratteristiche e si sviluppino attraverso verifiche sperimentali ed euristiche (come ad esempio quelle di Ma. e Or.) o l'identificazione di relazioni tra i dati (con prova procedurale come quella proposta da Fe.). L'approccio di generalizzazione per l'interpretazione del fenomeno in termini di analisi dei dati sembra svilupparsi quindi attraverso la generazione delle diverse ipotesi costituendo, di fatto, le componenti essenziali del processo argomentativo.

In sintesi, le esperienze didattiche descritte risultano essere efficaci nel perseguire quegli obiettivi trasversali che vedono gli alunni come cittadini capaci di produrre ipotesi, saper riconoscere ipotesi

2. La scuola secondaria in Italia si divide in scuola secondaria di primo grado, che dura tre anni e corrisponde ai primi tre anni di scuola media nel Canton Ticino, e scuola secondaria di secondo grado, che dura cinque anni e corrisponde all'ultimo anno di scuola media e alla scuola media superiore o scuole professionali nel Canton Ticino. 
vere e, soprattutto, comprendere perché esse sono vere (o false) sostenendo quindi il proprio punto di vista con argomenti pertinenti e confrontandosi con i punti di vista degli altri (questo emerge dagli interventi degli alunni, si vedano ad esempio quelli di Au.).

\section{Bibliografia}

Bartolini Bussi, M., Boni, M., \& Ferri, F. (1995). Interazione sociale e conoscenza a scuola: La discussione matematica. Modena: CDE.

Ben-Zvi, D. (2014). Data handling and statistics teaching and learning. In S. Lerman (Ed.), Encyclopedia of mathematics education (pp. 137-140). Dordrecht: Springer.

Boero, P., \& Ferrero, E. (1995). II gioco delle ipotesi nell'insegnamento-apprendimento della matematica nella scuola dell'obbligo: una ricerca in corso. L'insegnamento della matematica e delle scienze integrate, v. $18 \mathrm{~A}-18 \mathrm{~B}$

Gattuso, L., \& Ottaviani, M. G. (2011). Complementing mathematical thinking and statistical thinking in school Mathematics. In C. Batanero, G. Burrill \& C. Reading (Eds.), Teaching Statistics in School Mathematics-Challenges for Teaching and Teacher Education, (Vol 14, pp. 121-132). Dordrecht, Springer, New Icmi Study Series.

Gravemeijer, K. (1999). How emergent models may foster the constitution of formal mathematics. Mathematical Thinking and Learning, 1(2), 155-177.

Kranendonk, H. (2006). A statistical study of generations. In G. Burrill (Ed.), Thinking and reasoning with data and chance. NCTM 2006 yearbook (pp. 103-116). Reston, VA: NCTM

MIUR (2012). Indicazioni Nazionali per il curricolo della scuola dell'infanzia e del primo ciclo di istruzione. Disponibile in: http://www.indicazioninazionali.it/wp-content/uploads/2018/08/Indicazioni_Annali_Definitivo. pdf (consultato il 22.09.2020)

MIUR (2018). Indicazioni nazionali e nuovi scenari. Disponibile in: http://www.miur.gov.it/documents/20182/0/ Indicazioni+nazionali+e+nuovi+scenari/3234ab16-1f1d-4f34-99a3-319d892a40f2 (consultato il 22.09.2020).

Ottaviani, M. G. (2008). Statistica e matematica a scuola: due discipline e un solo insegnamento. Confronto culturale e opportunità interdisciplinare. Induzioni, 36, 17-38.

Sabena, C., Ferri, F., Martignone, F., \& Robotti, E. (2019). Insegnare e apprendere matematica nella scuola dell'infanzia e primaria. Milano: Mondadori.

Skemp, R. R. (1976). Relational understanding and instrumental understanding. Mathematics Teaching, 77, $20-27$.

U.M.I. (2001). Matematica 2001- Materiali per un muovo curricolo di matematica con suggerimenti per attività e prove di verifica. Disponibile in: http://www.umi-ciim.it/materiali-umi-ciim/primo-ciclo/ (consultato il 22.09.2020).

Vergnaud, G. (1992). Teoria dei campi concettuali, La matematica e la sua didattica, 1, 4-19. (Titolo originale: La théorie des champs conceptuels pubblicato nel 1990). 